\title{
The equation of motion phonon method and its application in the neutron rich oxygen region
}

\author{
G. De Gregorio ${ }^{1,2, *}, F$ Knapp $^{3}, N$. Lo Iudice ${ }^{2,4}$, and $P$. Veselý ${ }^{1}$ \\ ${ }^{1}$ Nuclear Physics Institute, Czech Academy of Sciences, 25068 Řež, Czech Republic \\ ${ }^{2}$ INFN Sezione di Napoli, 80126 Napoli, Italy \\ ${ }^{3}$ Faculty of Mathematics and Physics, Charles University, 11636 Prague, Czech Republic \\ ${ }^{4}$ Università di Napoli Federico II, 80126 Napoli, Italy
}

\begin{abstract}
An equation of motion phonon method, developed for even-even nuclear systems and extended to odd nuclei, is applied to ${ }^{22} \mathrm{O}$ and to its odd neighbors ${ }^{23} \mathrm{O}$ and ${ }^{23} \mathrm{~F}$. A calculation using the chiral potential $\mathrm{NNLO}_{\text {opt }}$ is carried out in a space encompassing up to two phonons. The computed dipole cross section in ${ }^{22} \mathrm{O}$ and the spectra of ${ }^{22} \mathrm{O}$ and ${ }^{23} \mathrm{O}$ are in a satisfactory agreement with the experimental data. However, the calculation describes poorly the spectrum of ${ }^{23} \mathrm{~F}$. This discrepancy originates from the strong coupling between the odd proton and the ${ }^{22} \mathrm{O}$ phonons of neutron nature. This coupling pushes down in energy several states enhancing the level density at low energy. We suggest that a viable route for the solution of this problem could be the inclusion of the three-body interaction using the new chiral potential $\mathrm{NNLO}_{\text {sat }}$.
\end{abstract}

\section{Introduction}

In the last decades, several experiments have been carried out to study the spectroscopic properties of neutron rich isotopes in the oxygen region. In particular, the occurrence of new magic numbers, $N=14$ and $N=16$, the limit of the neutron dripline and the pygmy dipole resonance (PDR) have been subjects of extensive studies [1-15].

The large amount of data produced has stimulated several theoretical investigations. Many coupled cluster calculations have been performed to study bulk properties and low-energy levels, most of them using $\mathrm{NN}+3 \mathrm{~N}$ chiral forces derived from the effective field theory [16-22].

Three-body forces have been used also within a manybody perturbation theory calculation [23], a shell model calculation [24], and a self-consistent Green's function theory approach [25].

The equation of motion phonon method (EMPM) has been also used to study the nuclei in this region. The method was first formulated for even-even nuclei in the particle-hole (p-h) scheme [26-28] and, then, in terms of Hartree-Fock-Bogoliubov (HFB) quasiparticles (qp) [29]. It derives a set of equations yielding a basis of orthonormal multiphonon states, built of phonons obtained in TammDancoff approximation (TDA), and, then, solves the full eigenvalue problem in the space spanned by such a basis. It takes the Pauli principle into full account and does not rely on any approximation. It has been adopted to study spectra and dipole responses of light and heavy even-even nuclei [30-33].

More recently it has been also extended to odd nuclei [34-36]. An orthonormal basis of states composed of a va-

\footnotetext{
*e-mail: degregorio@na.infn.it
}

lence particle coupled to $n$-phonon states $(n=0,1,2, \ldots)$, describing the excitations of a doubly magic core, is produced by a set of equations of motion and, then, employed for the solution of the full eigenvalue problem.

Here we report on the application of the EMPM to study the low- and high-energy spectroscopic properties of ${ }^{22} \mathrm{O}$ and its adjacent nuclei ${ }^{23} \mathrm{O}$ and ${ }^{23} \mathrm{~F}$ [37]. The comparison of the results with the experimental data and the analysis of the phonon composition contributes to disclose the nature of the states and shows the limits of the adopted potential.

\section{The Equation of Motion Phonon Method for even-even nuclei}

Let us consider the Hamiltonian

$$
H=H_{0}+V \text {. }
$$

$H_{0}$ is the one-body term

$$
H_{0}=\sum_{r}[r]^{1 / 2} \epsilon_{r}\left(a_{r}^{\dagger} \times b_{r}\right)^{0}
$$

where $a_{r}^{\dagger}=a_{x_{r} j_{r} m_{r}}^{\dagger}\left(b_{r}=(-)^{j_{r}+m_{r}} a_{x_{r} j_{r} m_{r}}\right)$ creates (annihilates) a particle of energy $\epsilon[r]$ stands for $[r]=2 j_{r}+1$ and the symbol $\times$ denotes angular momentum coupling. $V$ is a two-body potential

$$
V=\frac{1}{4} \sum_{r s q t \sigma}[\sigma]^{1 / 2} F_{r s q t}^{\sigma}\left[\left(a_{r}^{\dagger} \times b_{s}\right)^{\sigma} \times\left(a_{q}^{\dagger} \times b_{t}\right)^{\sigma}\right]^{0},
$$

where $F^{\sigma}$ is derived from the two-body potential $V^{\Omega}$ through the Pandya transformation

$$
F_{r s q t}^{\sigma}=\sum_{\Omega}[\omega](-)^{(r+t-\sigma-\Omega)} W(r s q t ; \sigma \Omega) V_{r s t q}^{\Omega} .
$$


Here $W(r s q t ; \sigma \Omega)$ are Racah coefficients.

The primary goal of the method is to derive a basis of $n$-phonon correlated states of the form

$$
\begin{aligned}
\left|\beta_{n}\right\rangle & =\sum_{\lambda \alpha_{n-1}} C_{\lambda \alpha_{n-1}}^{\beta_{n}}\left|\left(\lambda \times \alpha_{n-1}\right)^{\beta_{n}}\right\rangle \\
& =\sum_{\lambda \alpha_{n-1}} C_{\lambda \alpha_{n-1}}^{\beta_{n}}\left\{O_{\lambda}^{\dagger} \times\left|\alpha_{n-1}\right\rangle\right\}^{\beta_{n}},
\end{aligned}
$$

of energy $E_{\beta_{n}}$, where

$$
O_{\lambda}^{\dagger}=\sum_{p h} c_{p h}^{\lambda}\left(a_{p}^{\dagger} \times b_{h}\right)^{\lambda}
$$

is the p-h TDA phonon operator of energy $E_{\lambda}$ acting on the $(n-1)$-phonon basis states $\mid \alpha_{n-1}>$, assumed to be known. The operators $a_{p}^{\dagger}=a_{x_{p} j_{p} m_{p}}^{\dagger}$ and $b_{h}=(-)^{j_{h}+m_{h}} a_{x_{h} j_{h}-m_{h}}$ create a particle and a hole of energies $\epsilon_{p}$ and $-\epsilon_{h}$, respectively.

As illustrated in Ref. [28], we start with the equations of motion

$$
\left\langle\beta_{n}\left\|\left[H, O_{\lambda}^{\dagger}\right]\right\| \alpha_{n-1}\right\rangle=\left(E_{\beta_{n}}-E_{\alpha_{n-1}}\right) X_{\lambda \alpha_{n-1}}^{\beta_{n}},
$$

where

$$
\begin{aligned}
X_{\lambda \alpha_{n-1}}^{\beta_{n}} & =\left\langle\beta_{n}\left\|O_{\lambda}^{\dagger}\right\| \alpha_{n-1}\right\rangle \\
& =\left[\beta_{n}\right]^{1 / 2} \sum_{\lambda^{\prime} \alpha_{n-1}^{\prime}} \mathcal{D}_{\lambda \alpha_{n-1} \lambda^{\prime} \alpha_{n-1}^{\prime}}^{\beta_{n}} C_{\lambda^{\prime} \alpha_{n-1}^{\prime}}^{\beta_{n}} .
\end{aligned}
$$

Here $\left[\beta_{n}\right]=2 J_{\alpha_{n}}+1$, a notation which will be used throughout the paper, and

$$
\mathcal{D}_{\lambda \alpha_{n-1} \lambda^{\prime} \alpha_{n-1}^{\prime}}^{\beta_{n}}=\left\langle\left(\lambda \times \alpha_{n-1}\right)^{\beta_{n}} \mid\left(\lambda^{\prime} \times \alpha_{n-1}^{\prime}\right)^{\beta_{n}}\right\rangle
$$

is the overlap or metric matrix which reintroduces the exchange terms among different phonons and, therefore, reestablishes the Pauli principle. Its expression can be found in [28]. From now on we will omit the subscript $n$ when acting within a $n$-phonon subspace.

After expanding the commutator in the Eq. (7) and expressing the amplitudes $X$ in terms of the coefficients $C_{\lambda \alpha}^{\beta}$ through Eq. (8), we obtain

$$
\begin{array}{r}
\sum_{\lambda_{1} \alpha_{1} \lambda^{\prime} \alpha^{\prime}}\left(\left(E_{\lambda}+E_{\alpha}-E_{\beta}\right) \delta_{\lambda \lambda_{1}} \delta_{\alpha \alpha_{1}}+\mathcal{V}_{\lambda \alpha \lambda_{1} \alpha_{1}}^{\beta}\right) \\
\times \mathcal{D}_{\lambda_{1} \alpha_{1} \lambda^{\prime} \alpha^{\prime}}^{\beta} C_{\lambda^{\prime} \alpha^{\prime}}^{\beta}=0,
\end{array}
$$

where $\mathcal{V}_{\lambda \alpha \lambda^{\prime} \alpha^{\prime}}^{\beta}$ is the phonon-phonon potential whose expression can be found in [28].

Equation (10) represents an eigenvalue equation in the over-complete basis $\left|(\lambda \times \alpha)^{\beta}\right\rangle$ within the $n$-phonon core subspace. The redundant states are eliminated following the procedure outlined in Refs. [26, 27], based on the Cholesky decomposition method. It allows to extract a basis of linearly independent states spanning the physical subspace to obtain a non singular eigenvalue equation whose iterative solution yields a basis of orthonormal correlated $n$-phonon states of the form (5).

The eigenvalue problem in such a basis is given by

$$
\sum_{n^{\prime} \beta_{n^{\prime}}}\left[\left(E_{\alpha_{n}}-\mathcal{E}_{v}\right) \delta_{n n^{\prime}} \delta_{\alpha_{n} \beta_{n^{\prime}}}+\mathcal{V}_{\alpha_{n} \beta_{n^{\prime}}}\right] C_{\beta_{n^{\prime}}}^{(v)}=0,
$$

where $\mathcal{V}_{\alpha_{n} \beta_{n^{\prime}}}$ are non vanishing for $n^{\prime}=n+1$ and $n^{\prime}=n+2$. The solution of the above eigenvalue equation yields the eigenvalues $\mathcal{E}_{v}$ and the eigenstates

$$
\left|\Psi_{v}\right\rangle=\sum_{n \alpha_{n}} C_{\alpha_{n}}^{(v)}\left|\alpha_{n}\right\rangle
$$

The wavefunctions (12) can be used to compute the transition amplitudes of a multipole operator

$$
\begin{array}{r}
\left\langle\Psi_{v^{\prime}}\|\mathcal{M}(\lambda)\| \Psi_{v}\right\rangle= \\
\sum_{\left(n \alpha_{n}\right)\left(n^{\prime} \beta_{n^{\prime}}\right)} C_{\alpha_{n}}^{(v)} C_{\beta_{n^{\prime}}}^{\left(\nu^{\prime}\right)}\left\langle\beta_{n^{\prime}}\|\mathcal{M}(\lambda)\| \alpha_{n}\right\rangle .
\end{array}
$$

Here the matrix elements of $\mathcal{M}(\lambda)$ between multiphonon states are

$$
\begin{array}{r}
\left\langle\beta_{n^{\prime}}\|\mathcal{M}(\lambda)\| \alpha_{n}\right\rangle=[\lambda]^{-1 / 2}\left[\delta_{n^{\prime} n} \mathcal{M}_{\alpha \beta}^{(n)}(\lambda)+\right. \\
+\sum_{x} \mathcal{M}(0 \rightarrow x \lambda)\left(\delta_{n^{\prime}(n+1)} X_{(x \lambda) \alpha_{n}}^{\beta_{n+1}}\right. \\
\left.\left.+(-)^{v^{\prime}-v} \delta_{n^{\prime}(n-1)} X_{(x \lambda) \beta_{n-1}}^{\alpha_{n}}\right)\right],
\end{array}
$$

where

$$
\begin{aligned}
\mathcal{M}(0 & \rightarrow x \lambda)=<x \lambda\|\mathcal{M}(\lambda)\| 0>= \\
& =\sum_{p h} c_{p h}^{(x \lambda)}<p\|\mathcal{M}(\lambda)\| h>
\end{aligned}
$$

is the TDA transition amplitude and

$$
\mathcal{M}_{\alpha \beta}^{(n)}(\lambda)=\sum_{r s}\langle r\|\mathcal{M}(\lambda)\| s\rangle\left\langle\alpha\left\|\left(a_{r}^{\dagger} \times b_{s}\right)^{\lambda}\right\| \beta\right\rangle
$$

is the scattering term between states with the same number of phonons $\left(n^{\prime}=n\right)$.

\section{The Equation of Motion Phonon Method for odd nuclei}

An analogous procedure is adopted to generate an orthonormal multiphonon particle-core basis for a valence nucleon external to a doubly magic core, and then, to solve the full eigenvalue problem.

The basis states $|v\rangle$ of spin $v$ have the form

$$
|v\rangle=\sum_{p \alpha} C_{p \alpha}^{v}\left|(p \times \alpha)^{v}\right\rangle=\sum_{p \alpha} C_{p \alpha}^{v}\left(a_{p}^{\dagger} \times|\alpha\rangle\right)^{v},
$$

where $|\alpha\rangle$ are the $n$-phonon core states (5).

In close analogy with the even nuclei we start with

$$
\left\langle\alpha\left\|\left[b_{p}, H\right]^{p}\right\| v\right\rangle=\left(E_{v}-E_{\alpha}\right) X_{p \alpha}^{(v)}
$$

where

$$
X_{p \alpha}^{(v)}=\left\langle\alpha\left\|b_{p}\right\| v\right\rangle=\sum_{p_{1} \alpha_{1}} \mathcal{D}_{p^{\prime} \alpha^{\prime} p_{1} \alpha_{1}}^{(v)} C_{p_{1} \alpha_{1}}^{v}
$$

and

$$
\mathcal{D}_{p \alpha p^{\prime} \alpha^{\prime}}^{(v)}=\left\langle\left(p^{\prime} \times \alpha^{\prime}\right)^{v} \mid(p \times \alpha)^{v}\right\rangle
$$


is the overlap matrix which reintroduces the exchange terms among the odd particle and the $n$-phonon states and, therefore, re-establishes the Pauli principle.

After expanding the commutator and expressing $X$ in terms of $C_{p_{1} \alpha_{1}}^{v}$ through the Eq. (19) we obtain

$$
\begin{array}{r}
\sum_{p^{\prime} \alpha^{\prime} p_{1} \alpha_{1}}\left\{\left(\epsilon_{p}+E_{\alpha}-E_{v}\right) \delta_{p p^{\prime}} \delta_{\alpha \alpha^{\prime}}+\mathcal{V}_{p \alpha p^{\prime} \alpha^{\prime}}^{(v)}\right\} \\
\times \mathcal{D}_{p^{\prime} \alpha^{\prime} p_{1} \alpha_{1}}^{(v)} C_{p_{1} \alpha_{1}}^{v}=0 .
\end{array}
$$

where $\mathcal{V}_{p \alpha p^{\prime} \alpha^{\prime}}^{(v)}$ is the particle-phonon interaction.

Equation (21) represents an eigenvalue equation in the over-complete basis $\left|(p \times \alpha)^{v}\right\rangle$ within the n-phonon particle-core subspace. Following the same procedure adopted for even nuclei, based on the Cholesky decomposition method, we extract a basis of linearly independent states and obtain a non-singular eigenvalue equations whose iterative solution yields the orthonormal particlecore states $\left|v_{n}\right\rangle$. This states can be used to diagonalize the full Hamiltonian

$$
\sum_{v_{n^{\prime}}^{\prime}}\left\{\left(E_{v_{n}}-\mathcal{E}_{v}\right) \delta_{v_{n} v_{n^{\prime}}^{\prime}}+\mathcal{V}_{v_{n} v_{n^{\prime}}^{\prime}}^{(v)}\right\} C_{v_{n^{\prime}}^{\prime}}^{(v)}=0
$$

where $\mathcal{V}_{v_{n} v_{n^{\prime}}^{\prime}}^{(v)}$ is non vanishing only for $n^{\prime} \neq n$. Eq. (22) yields all the eigenvalues allowed by the space dimensions and the eigenfunctions

$$
\left|\Psi_{v}\right\rangle=\sum_{v_{n}} C_{v_{n}}^{v}\left|v_{n}\right\rangle
$$

where $\left|v_{n}\right\rangle$ is given by Eq. (17).

Using the wavefunctions (23), we get the transition amplitudes

$$
\left\langle\Psi_{v^{\prime}}\|\mathcal{M}(\lambda)\| \Psi_{v}\right\rangle=\sum_{v_{n} v_{n^{\prime}}^{\prime}} C_{v_{n}}^{v} C_{v_{n^{\prime}}^{\prime}}^{v^{\prime}}\left\langle v_{n^{\prime}}^{\prime}\|\mathcal{M}(\lambda)\| v_{n}\right\rangle
$$

where

$$
\left\langle v_{n^{\prime}}^{\prime}\|\mathcal{M}(\lambda)\| v_{n}\right\rangle=[v]^{1 / 2} \sum_{p \alpha_{n}} C_{p \alpha_{n}}^{v_{n}} \mathcal{M}_{p \alpha_{n}}^{\left(v_{n} v_{n^{\prime}}^{\prime}\right)}(\lambda) .
$$

For $n^{\prime}=n$ we have

$$
\begin{gathered}
\mathcal{M}_{p \alpha_{n}}^{\left(v_{n} v_{n}^{\prime}\right)}(\lambda)=\sum_{p^{\prime}} W\left(\lambda p^{\prime} v \alpha_{n} ; p v^{\prime}\right)\left\langle p^{\prime}\left\|\mathcal{M}_{\lambda}\right\| p\right\rangle X_{p^{\prime} \alpha_{n}}^{\left(v_{n}^{\prime}\right)} \\
\quad+\sum_{\alpha_{n}^{\prime}} W\left(p \alpha_{n} v^{\prime} \lambda p ; v \alpha_{n}^{\prime}\right)\left\langle\alpha_{n}^{\prime}\|\mathcal{M}(\lambda)\| \alpha_{n}\right\rangle X_{p \alpha_{n}^{\prime}}^{\left(v_{n}^{\prime}\right)}
\end{gathered}
$$

For $n^{\prime}=n+1$ we get

$$
\begin{aligned}
\mathcal{M}_{p \alpha_{n}}^{\left(v_{n} v_{n+1}^{\prime}\right)}(\lambda)= & \sum_{\beta_{n+1}} W\left(\lambda \alpha_{n} v^{\prime} p ; \beta_{n+1} v\right) X_{p \beta_{n+1}}^{\left(v_{n+1}^{\prime}\right)} \\
& \times \sum_{x} \mathcal{M}(0 \rightarrow[x \lambda]) X_{(x \lambda) \alpha_{n}}^{\left(\beta_{n+1}\right)}
\end{aligned}
$$

The transition amplitude for $n^{\prime}=n-1$ can be deduced from the one for $n^{\prime}=n+1$.

\section{Calculations and results}

We used a Hamiltonian composed of an intrinsic kinetic operator $T_{\text {int }}$ plus the $\mathrm{NNLO}_{\text {opt }}$ optimized chiral potential [38] to generate the HF basis in a configuration space encompassing all the harmonic oscillator major shells up to $N_{\max }=15$.

The TDA phonons are derived from a subset of HF states corresponding to $\mathrm{N}=7$. Their structure does not change if we use the full HF space. The spurious $J^{\pi}=1^{-}$ TDA components induced by the center of mass (CM) motion have been removed by resorting to a Gramm-Schmidt orthogonalization procedure discussed in Ref. [39].

All the TDA phonons with dominant $0-\hbar \omega$ and $1-\hbar \omega$ components are the constituents of the two-phonon states in ${ }^{22} \mathrm{O}$. These, with the TDA phonons, are the core states coupled to the odd particle for generating the multiphonon basis of ${ }^{23} \mathrm{O}$ and ${ }^{23} \mathrm{~F}$.

\subsection{Spectra of ${ }^{22} \mathrm{O},{ }^{23} \mathrm{O}$ and ${ }^{23} \mathrm{~F}$}

The EMPM and the experimental spectra are compared to one another in Fig. 1.

In ${ }^{22} \mathrm{O}$ we obtain a one to one correspondence between the computed low-lying positive parity states and the experimental levels [12]. The negative parity states are estimated to be above the neutron decay threshold consistently with experiments.

The mixing of the HF vacuum with the two-phonon components is very modest. In fact, the total wave function is composed of a large number of two-phonon states of different multipolarities, all having small amplitudes, whose overall contribution amounts to $8.5 \%$.

The excited states have an almost pure $n-$ phonon character. The lowest $2_{1}^{+}$and $3_{1}^{+}$are almost pure neutron TDA phonons arising from single $\mathrm{p}-\mathrm{h}$ excitation in the $s d$ shell.

The subsequent triplet of states, $\left\{0_{2}^{+}, 2_{2}^{+}, 4_{2}^{+}\right\}$is of twophonon nature. These states are composed of the low-lying $2_{1}^{+}$and $3_{1}^{+}$phonons and, therefore, have a neutron nature as well. Their composition suggests an harmonic character of the triplet, which, however, is confirmed only by the experimental spectrum.

The low-lying negative parity states appear at $\sim 7 \mathrm{MeV}$ and have a one-phonon nature. They arise mainly from exciting a neutron from $0 d_{5 / 2}$ to $1 p_{1 / 2}$ or $1 p_{3 / 2}$.

The spectrum of ${ }^{23} \mathrm{O}$ determined experimentally [10] is composed of two positive parity $5 / 2^{+}$and $3 / 2^{+}$levels and a level, probably of negative parity, at higher energy. All of them are above the neutron decay threshold (Fig. 1).

The computed levels are also above such a threshold. The positive parity ones are close in energy to the corresponding experimental levels, and the negative parity states are compatible in spin and energy with the corresponding detected level.

As in ${ }^{22} \mathrm{O}$, all the states have substantially a single $n$-phonon structure. While the $3 / 2_{1}^{+}$and the lowest negative parity states have a single particle nature, the first excited $5 / 2_{1}^{+}$is a particle-phonon state resulting, almost entirely, from coupling the $1 / 2_{1}^{+}$particle to the $3_{1}^{+}$phonon. This component accounts for $\sim 97 \%$ of the state. 


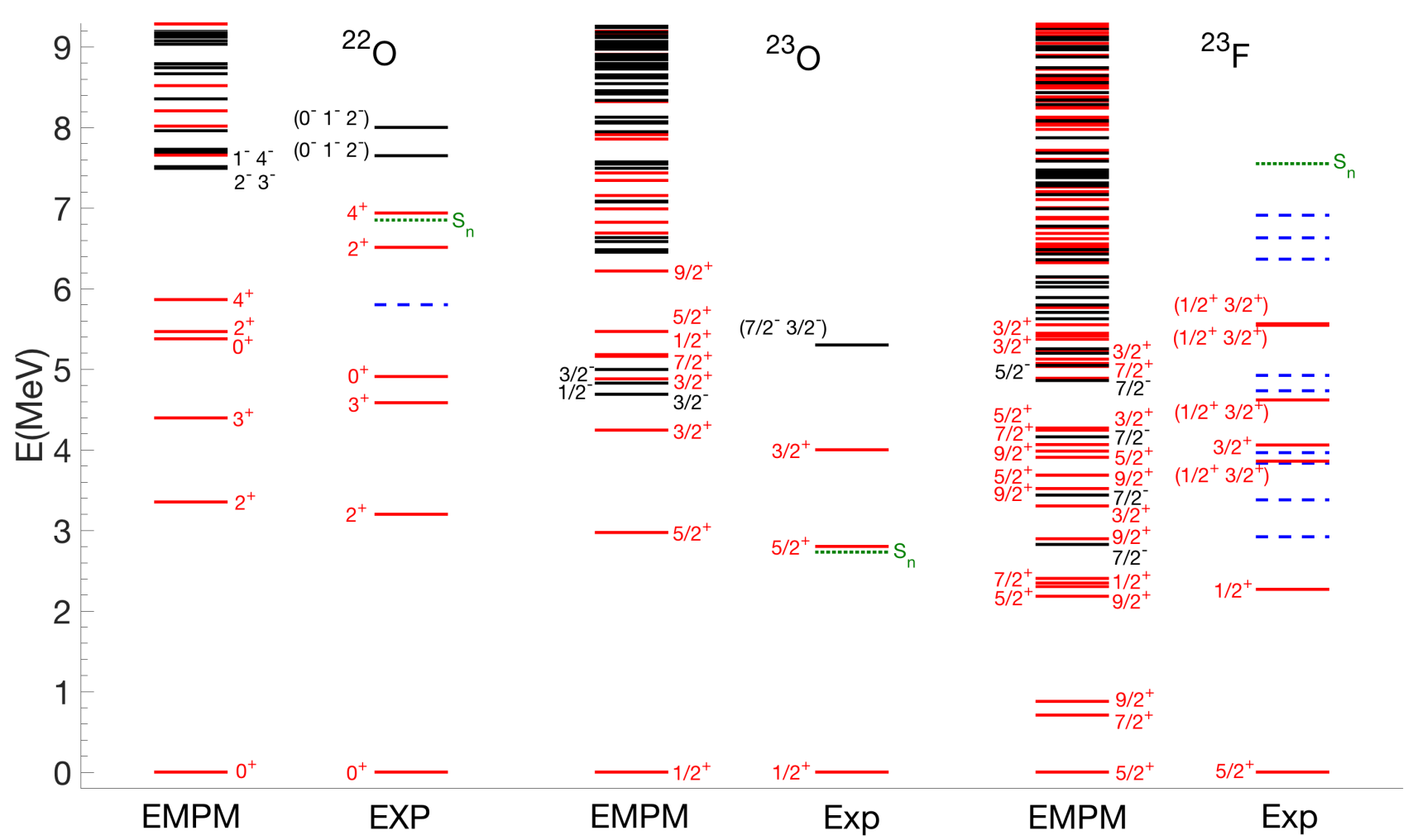

Figure 1. (Color online) Theoretical versus experimental $[4,5,10,12]$ spectra of ${ }^{22} \mathrm{O},{ }^{23} \mathrm{O}$ and ${ }^{23} \mathrm{~F}$. The dashed lines denote levels of unknown spin and parity. The dotted lines indicate the neutron decay thresholds.

In the energy interval $\sim 4.8-6.2 \mathrm{MeV}$ the calculation yields a quintuplet of positive parity states $\left\{1 / 2_{2}^{+}, 3 / 2_{2}^{+}, 5 / 2_{2}^{+}, 7 / 2_{1}^{+}, 9 / 2_{1}^{+}\right\}$arising from the coupling of the $1 / 2_{1}^{+}$neutron particle to the low-lying two-phonon harmonic triplet of the ${ }^{22} \mathrm{O}$ already discussed (Fig. 1). Thus, the computed ${ }^{23} \mathrm{O}$ level scheme keeps memory of the harmonic nature of the spectrum of the ${ }^{22} \mathrm{O}$.

The experimental spectrum of ${ }^{23} \mathrm{~F}[4,5]$ is much richer compared to ${ }^{23} \mathrm{O}$, and is described poorly within the EMPM. In fact, several theoretical levels occur in the lowenergy region without an experimental counterpart.

The reason of such a high density of levels at low energy is the too strong proton-phonon coupling. The origin of such a strong coupling resides in the neutron dominance of the low-energy phonons which arise entirely from the excitations within the neutron $s d$ shell.

\subsection{Dipole response in ${ }^{22} \mathrm{O}$}

As shown in Fig. 2, the theoretical dipole cross section of the ${ }^{22} \mathrm{O}$ is in qualitatively good agreement with the experimental data [13]. The ground state correlation induced by the two-phonon states shifts upward in energy by $\sim 2 \mathrm{MeV}$ the calculated cross section without changing its shape. The highest peak occurs at $\sim 30 \mathrm{MeV}$ and is out of the experimental region.

The integrated cross section up to $\sim 25 \mathrm{MeV}$ exhausts $\sim 30 \%$ of the TRK sum rule. This value is in agreement, within the errors, with the experimental sum. However,

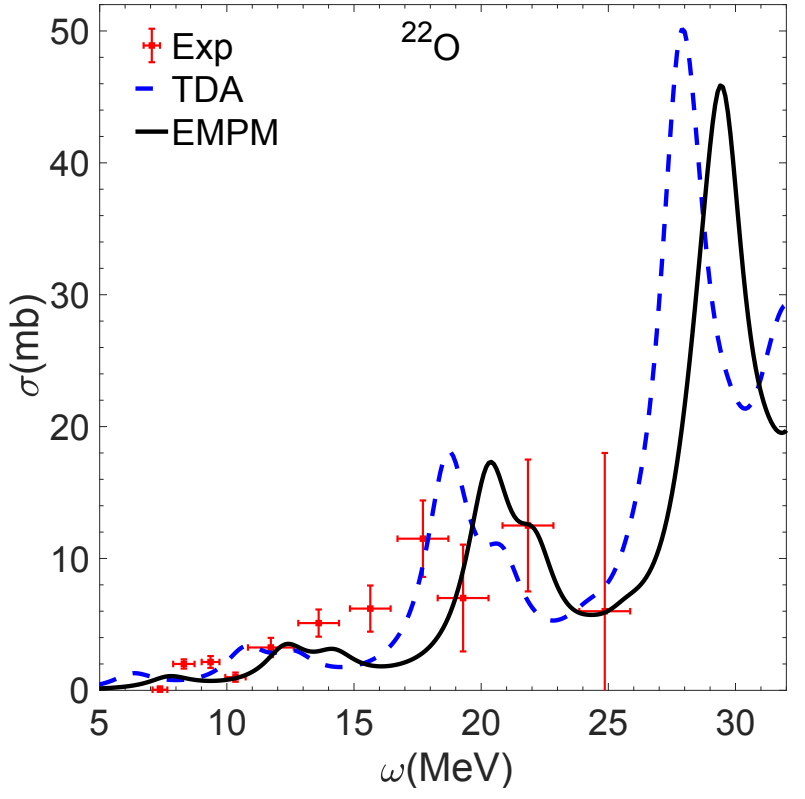

Figure 2. Theoretical versus experimental [13] $E 1$ cross section in ${ }^{22} \mathrm{O}$. A Lorentzian of width $\Delta=2 \mathrm{MeV}$ is used.

the largest contribution comes from the strength at higher energy. In fact, the total sum up to $\sim 40$ accounts for $\sim$ $125 \%$

At low energy $\sim 7-15 \mathrm{MeV}$, the cross section exhibit small humps which can be associated to the PDR. In fact, 


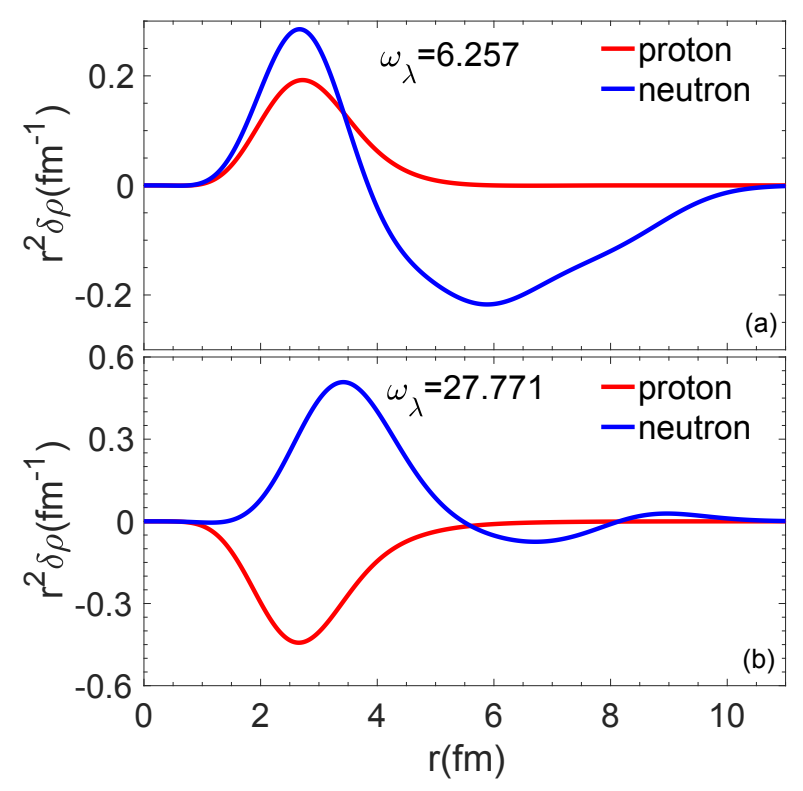

Figure 3. Transition densities for the lowest $1^{-}$state (a) and one falling in the region of the GDR (b)

as confirmed by the transition densities plotted in Fig. 3, the neutron skin oscillates against the core at low energy (Fig. 3 (a)) while proton and neutron fluids oscillate in opposition of phases in the GDR region (Fig. 3 (b)).

\section{Conclusions}

The EMPM describes fairly well the spectra of ${ }^{22} \mathrm{O}$ and ${ }^{23} \mathrm{O}$. In ${ }^{22} \mathrm{O}$ there is a one to one correspondence between theoretical and experimental positive parity low-energy states. The lowest $2_{1}^{+}$and $3_{1}^{+}$are almost pure neutron TDA phonons arising from single $\mathrm{p}-\mathrm{h}$ excitation within the $s d$ shell. These states are the constituents of an harmonic triplet of states, $\left\{0_{2}^{+}, 2_{2}^{+}, 4_{1}^{+}\right\}$, falling in the energy region $\sim 5-6 \mathrm{MeV}$.

The negative parity states have spin and energy compatible with the experimental levels, occur at higher energy and arise from the excitation of $0 d_{5 / 2}$ neutron hole to the $p f$ particle shell.

In ${ }^{23} \mathrm{O}$, the few measured levels are well reproduced. They are shown to have a dominant neutron character and to have a pure particle-phonon structure. In fact, the weak neutron-neutron interaction is ineffective in inducing a mixing among different $n$-phonon subspaces given the neutron nature of the low-lying phonons of ${ }^{22} \mathrm{O}$.

The neutron dominance of the latter phonons has an opposite effect on the ${ }^{23} \mathrm{~F}$. In fact, the proton-phonon coupling induced by the strongly attractive neutron-proton interaction is magnified by such a dominance pushes several states into the low-energy region thereby enhancing greatly the density of levels.

This mechanism explains why the description of the ${ }^{23} \mathrm{~F}$ spectrum is unsatisfactory.

A more moderate dominance of the low-lying neutron $\mathrm{p}$-h states would not only weaken the neutron-proton dom- inance of the particle-phonon coupling but also improve further the spectra of ${ }^{22} \mathrm{O}$ and ${ }^{23} \mathrm{O}$ by partly breaking their too harmonic character.

A possible recipe is to replace the adopted potential with the $\mathrm{NNLO}_{\text {sat }}$ [40]. This potential includes explicitly the three-body contribution and improves the description of binding energies and nuclear radii as well [41]. Preliminary calculations using such a potential in a harmonic oscillator space encompassing up to twelve major shells yield more compact HF level schemes. In particular, the gaps between the $(s d)$ and $(0 p)$ states is $\sim 13 \mathrm{MeV}$ for protons and $\sim 11 \mathrm{MeV}$ for neutrons, much smaller than the corresponding gaps produced by $\mathrm{NNLO}_{\text {opt }}$, $\sim 20 \mathrm{MeV}$. We feel therefore encouraged to pursue along this direction.

This work was partly supported by the Czech Science Foundation (Czech Republic), P203-13-07117S. Two of the authors (F. Knapp and P. Veselý) thank the INFN (Italy) for financial support. Highly appreciated was the access to computing and storage facilities provided by the Meta Centrum under the program LM2010005 and the CERIT-SC under the program Centre CERIT Scientific Cloud, part of the Operational Program Re- search and Development for Innovations, Reg. No. CZ.1.05/3.2.00/08.0144.

\section{References}

[1] P. Thirolf, B. Pritychenko, B. Brown, P. Cottle, M. Chromik, T. Glasmacher, G. Hackman, R. Ibbotson, K. Kemper, T. Otsuka, et al., Phys. Lett. B 485, 16 (2000)

[2] A. Ozawa, T. Kobayashi, T. Suzuki, K. Yoshida, and I. Tanihata, Phys. Rev. Lett. 84, 5493 (2000)

[3] E. Becheva, Y. Blumenfeld, E. Khan, D. Beaumel, J. M. Daugas, F. Delaunay, C.-E. Demonchy, A. Drouart, M. Fallot, A. Gillibert, et al., Phys. Rev. Lett. 96, 012501 (2006)

[4] S. Michimasa, S. Shimoura, H. Iwasaki, M. Tamaki, S. Ota, N. Aoi, H. Baba, N. Iwasa, S. Kanno, S. Kubono, et al., Phys. Lett. B 638, 146 (2006)

[5] C. S. Sumithrarachchi, D. J. Morrissey, B. A. Brown, A. D. Davies, D. A. Davies, M. Fancina, E. Kwan, P. F. Mantica, M. Portillo, Y. Shimbara, et al., Phys. Rev. C 75, 024305 (2007)

[6] A. Schiller, N. Frank, T. Baumann, D. Bazin, B. A. Brown, J. Brown, P. A. De Young, J. E. Finck, A. Gade, J. Hinnefeld, et al., Phys. Rev. Lett. 99, 112501 (2007)

[7] Z. Elekes, Z. Dombrádi, N. Aoi, S. Bishop, Z. Fülöp, J. Gibelin, T. Gomi, Y. Hashimoto, N. Imai, N. Iwasa, et al., Phys. Rev. Lett. 98, 102502 (2007)

[8] C. R. Hoffman, T. Baumann, D. Bazin, J. Brown, G. Christian, P. A. DeYoung, J. E. Finck, N. Frank, J. Hinnefeld, R. Howes, et al., Phys. Rev. Lett. 100, 152502 (2008)

[9] R. Kanungo, C. Nociforo, A. Prochazka, T. Aumann, D. Boutin, D. Cortina-Gil, B. Davids, M. Diakaki, 
F. Farinon, H. Geissel, et al., Phys. Rev. Lett. 102, 152501 (2009)

[10] C. R. Hoffman, T. Baumann, J. Brown, P. A. DeYoung, J. E. Finck, N. Frank, J. D. Hinnefeld, S. Mosby, W. A. Peters, W. F. Rogers, et al., Phys. Rev. C 83, 031303 (2011)

[11] E. Lunderberg, P. A. DeYoung, Z. Kohley, H. Attanayake, T. Baumann, D. Bazin, G. Christian, D. Divaratne, S. M. Grimes, A. Haagsma, et al., Phys. Rev. Lett 108, 142503 (2012)

[12] M. S. Basunia, Nuclear Data Sheets 127, 69 (2015)

[13] A. Leistenschneider, T. Aumann, K. Boretzky, D. Cortina, J. Cub, U. Datta Pramanik, W. Dostal, T. W. Elze, H. Emling, H. Geissel, et al., Phys. Rev. Lett. 86, 5442 (2001)

[14] E. Tryggestad, T. Aumann, T. Baumann, D. Bazin, J. R. Beene, Y. Blumenfeld, B. A. Brown, M. Chartier, M. L. Halbert, P. Heckman, et al., Phys. Lett. B 541, $52(2002)$

[15] E. Tryggestad, T. Baumann, P. Heckman, M. Thoennessen, T. Aumann, D. Bazin, Y. Blumenfeld, J. R. Beenec, T. A. Lewis, D. C. Radford, et al., Phys. Rev. C 67, 064309 (2003)

[16] J. R. Gour, P. Piecuch, M. Hjorth-Jensen, M. Wloch, and D. J. Dean, Phys. Rev. C 74, 024310 (2006)

[17] G. Hagen, T. Papenbrock, D. J. Dean, M. HjorthJensen, and B. Velamur Asokan, Phys. Rev. C 80, 021306(R) (2009)

[18] G. Hagen, T. Papenbrock, and M. Hjorth-Jensen, Phys. Rev. Lett. 104, 182501 (2010)

[19] O. Jensen, G. Hagen, M. Hjorth-Jensen, and J. S. Vaagen, Phys. Rev. C 83, 021305 (2011)

[20] O. Jensen, G. Hagen, M. Hjorth-Jensen, B. A. Brown, and A. Gade, Phys. Rev. Lett. 107, 032501 (2011)

[21] G. Hagen, M. Hjorth-Jensen, G. R. Jansen, R. Machleidt, and T. Papenbrock, Phys. Rev. Lett. 108, 242501 (2012)

[22] G. R. Jansen, J. Engel, G. Hagen, P. Navrátil, and A. Signoracci, Phys. Rev. Lett. 113, 142502 (2014)

[23] J. D. Holt, J. Menéndez, and A. Schwenk, Eur. Phys. J. A 49, 39 (2013)

[24] T. Otsuka, T. Suzuki, J. D. Holt, A. Schwenk, and Y. Akaishi, Phys. Rev. Lett. 105, 032501 (2010)
[25] A. Cipollone, C. Barbieri, and P. Navrátil, Phys. Rev. Lett. 111, 062501 (2013)

[26] F. Andreozzi, F. Knapp, N. Lo Iudice, A. Porrino, and J. Kvasil, Phys. Rev. C 75, 044312 (2007)

[27] F. Andreozzi, F. Knapp, N. Lo Iudice, A. Porrino, and J. Kvasil, Phys. Rev. C 78, 054308 (2008)

[28] D. Bianco, F. Knapp, N. Lo Iudice, F. Andreozzi, and A. Porrino, Phys. Rev. C 85, 014313 (2012)

[29] G. De Gregorio, F. Knapp, N. Lo Iudice, and P. Veselý, Phys Rev. C 93, 044314 (2016)

[30] D. Bianco, F. Knapp, N. Lo Iudice, F. Andreozzi, A. Porrino, and P. Veselý, Phys. Rev. C 86, 044327 (2012)

[31] F. Knapp, N. Lo Iudice, P. Veselý, F. Andreozzi, G. De Gregorio, and A. Porrino, Phys. Rev. C 90, 014310 (2014)

[32] F. Knapp, N. Lo Iudice, P. Veselý, F. Andreozzi, G. De Gregorio, and A. Porrino, Phys. Rev. C 92, 054315 (2015)

[33] G. De Gregorio, J. Herko, F. Knapp, N. Lo Iudice, and P. Veselý, Phys. Rev. C 95, 024306 (2017)

[34] G. De Gregorio, F. Knapp, N. Lo Iudice, and P. Veselý, Phys. Rev. C 94, 061301(R) (2016)

[35] G. De Gregorio, F. Knapp, N. Lo Iudice, and P. Veselý, Phys. Rev. C 95, 034327 (2017)

[36] G. De Gregorio, F. Knapp, N. Lo Iudice, and P. Veselý, Phys. Scr. 92, 074003 (2017)

[37] G. De Gregorio, F. Knapp, N. Lo Iudice, and P. Veselý, Phys. Rev. C 97, 034311 (2018)

[38] A. Ekström, G. Baardsen, C. Forssén, G. Hagen, M. Hjorth-Jensen, G. R. Jansen, R. Machleidt, W. Nazarewicz, T. Papenbrock, J. Sarich, et al., Phys. Rev. Lett. 110, 192502 (2013)

[39] D. Bianco, F. Knapp, N. Lo Iudice, P. Veselý, F. Andreozzi, G. De Gregorio, and A. Porrino, J. Phys. G: Nucl. Part. Phys. 41, 025109 (2014)

[40] A. Ekström, G. R. Jansen, K. A. Wendt, G. Hagen, T. Papenbrock, B. D. Carlsson, C. Forssén, M. HjorthJensen, P. Navrátil, and W. Nazarewicz, Phys. Rev. C 91, 051301 (2015)

[41] V. Lapoux, V. Soma', C. Barbieri, H. Hergert, J. D. Holt, and S. R. Stroberg, Phys. Rev. Lett. 117, 052501 (2016) 